\title{
Cardiovascular phenotypes in ventilated patients with COVID-19 acute respiratory distress syndrome
}

\author{
Bruno Evrard ${ }^{1,2}$, Marine Goudelin 1,2, Noelie Montmagnon ${ }^{1}$, Anne-Laure Fedou ${ }^{1,2}$, Thomas Lafon ${ }^{2,3,4}$ and \\ Philippe Vignon ${ }^{1,2,4,5,6^{*}}$ (D)
}

Keywords: Acute respiratory distress syndrome, COVID-19, Influenza, Human, Echocardiography, Echocardiography, Doppler

Approximately two-thirds of patients admitted to the intensive care unit (ICU) for coronavirus disease-19 (COVID-19) pneumonia present with the acute respiratory distress syndrome (ARDS) [1]. COVID-19-associated acute cardiac injury is frequently reported based on troponin and electrocardiographic changes [2], but its impact on cardiac function is yet unknown [3]. Accordingly, we sought to describe cardiovascular phenotypes identified using transesophageal echocardiography (TEE) in ventilated COVID-19 patients with ARDS and to compare them to those of patients with flu-induced ARDS.

All patients with confirmed COVID-19 who were mechanically ventilated for ARDS in our medical-surgical ICU underwent prospectively a TEE assessment during the first 3 days and whenever required by clinical events during ICU stay, as a standard of care. Similarly, all patients ventilated for flu-associated ARDS who underwent a TEE assessment over the last 2 years were retrospectively analyzed for comparison. Cardiovascular phenotypes were identified using previously reported TEE criteria [4]. Same applied for acute cor pulmonale (ACP) [5]. TEE studies were read by two independent experts who had no access to the cause of ARDS and examination date. Results are expressed as medians and 25th75th percentiles. Friedman ANOVA was used to compare quantitative parameters over time in COVID-19 patients, while Mann-Whitney $U$ test and Fisher's exact test were used for comparison of continuous and categorical variables, respectively, with flu patients. No use of previous value or interpolation rule was used in the presence of missing data.

Eighteen consecutive COVID-19 patients and $23 \mathrm{flu}$ patients (21 A-H1N1) were studied. COVID-19 patients were significantly older (70 [57-75] vs. 58 [49-64] years, $p=0.006$ ), less severe (SAPSII 34 [30-38] vs. 43 [32-54], $p=0.015$; SOFA $4[2-4]$ vs. $6[4-9], p<0.001)$, required less vasopressor support $(2 / 18[11 \%]$ vs. 10/23 [43\%], $p=0.038$, and had longer time lag between first symptoms and ICU admission, tracheal intubation, and TEE examination when compared to flu patients (Table 1).

\footnotetext{
* Correspondence: philippe.vignon@unilim.fr

${ }^{1}$ Medical-Surgical Intensive Care Unit, Dupuytren Teaching Hospital, 87000 Limoges, France

${ }^{2}$ Inserm CIC 1435, Dupuytren Teaching Hospital, 87000 Limoges, France

Full list of author information is available at the end of the article
} 
Table 1 Characteristics, presentation and outcome of ventilated patients with COVID-19 and flu-related ARDS

\begin{tabular}{|c|c|c|c|}
\hline & COVID-19 $(n=18)$ & Flu $(n=23)$ & $p$ value \\
\hline \multicolumn{4}{|l|}{ Patients' characteristics } \\
\hline Age, years & $70(57-75)$ & $58(49-64)$ & 0.006 \\
\hline Male (\%) & $12(67)$ & $12(52)$ & 0.524 \\
\hline $\mathrm{BMI}, \mathrm{kg} / \mathrm{m}^{2}$ & $29(26-32)$ & $29(25-34)$ & 0.519 \\
\hline Hypertension (\%) & $11(61)$ & $10(43)$ & 0.350 \\
\hline Diabetes mellitus (\%) & $4(22)$ & $3(13)$ & 0.679 \\
\hline Time from illness onset to ICU admission, days & $11(7-13)$ & $5(4-10)$ & 0.017 \\
\hline Time from illness onset to intubation, days & $12(8-15)$ & $6(4-10)$ & 0.002 \\
\hline Time from illness onset to echocardiography, days & $14(9-17)$ & $13(6-17)$ & 0.001 \\
\hline SAPS ॥ & $34(30-38)$ & $43(32-54)$ & 0.015 \\
\hline SOFA score & $4(2-4)$ & $6(4-9)$ & $<0.001$ \\
\hline \multicolumn{4}{|l|}{ Clinical presentation and treatment } \\
\hline ECG changes* (\%) & $1(5 \%)$ & $3(13 \%)$ & 0.618 \\
\hline Documented coinfection (\%) & $3(17)$ & $9(39)$ & 0.171 \\
\hline Septic shock (\%) & $0(\%)$ & $10(43)$ & - \\
\hline Vasopressor support (\%) & $2(11)$ & $10(43)$ & 0.038 \\
\hline Prone position (\%) & $10(56)$ & $14(61)$ & 1.000 \\
\hline Neuromuscular blockers (\%) & $17(94 \%)$ & $12(52 \%)$ & 0.005 \\
\hline \multicolumn{4}{|l|}{ Biology on admission } \\
\hline Troponin I (ng/L) & $73(51-94)$ & $53(37-66)$ & 0.020 \\
\hline Lactate, $\mathrm{mmol} / \mathrm{L}$ & $1.17(0.89-1.57)$ & $1.51(1.02-2.54)$ & 0.143 \\
\hline Creatinine, $\mu \mathrm{mol} / \mathrm{L}$ & $58(42-87)$ & $88(59-160)$ & 0.021 \\
\hline Prothombine time, $\%$ & $87(78-96)$ & $87(71-101)$ & 0.979 \\
\hline AST, U/L & $55(27-71)$ & $107(46-203)$ & 0.020 \\
\hline $\mathrm{ALT}, \mathrm{U} / \mathrm{L}$ & $37(27-65)$ & $45(27-115)$ & 0.527 \\
\hline $\mathrm{CPK}, \mathrm{U} / \mathrm{L}$ & $72(34-103)$ & $419(180-2456)$ & $<0.001$ \\
\hline White blood cell count, G/L & $7.98(6.61-11.25)$ & $5.96(4.02-8.05)$ & 0.003 \\
\hline Lymphocyte count, G/L & $0,78(0.55-1.05)$ & $0.75(0.47-1.13)$ & 0.770 \\
\hline Eosinophils count, G/L & $0.02(0.02-0.09)$ & $0.01(0.00-0.01)$ & 0.094 \\
\hline Platelet count, G/L & $318(218-425)$ & $172(153-225)$ & $<0.001$ \\
\hline Hemoglobin, g/dl & $11.2(10.2-12.3)$ & $13.1(11.6-14.2)$ & 0.007 \\
\hline \multicolumn{4}{|l|}{ Respiratory parameters } \\
\hline $\mathrm{PaO}_{2} / \mathrm{FiO}_{2}$ & $130(81-217)$ & $70(62-100)$ & $<0.001$ \\
\hline Arterial pH & $7.35(7.29-7.45)$ & $7.32(7.23-7.41)$ & 0.121 \\
\hline $\mathrm{PaCO}_{2}, \mathrm{mmHg}$ & $44(33-51)$ & $47(36-60)$ & 0.430 \\
\hline $\mathrm{RR}$, breaths/min & $24(22-27)$ & $25(24-28)$ & 0.139 \\
\hline Tidal volume, $\mathrm{mL} / \mathrm{kg}$ & $5.2(4.5-6.2)$ & $5.3(4.0-6.1)$ & 0.885 \\
\hline PEEP, $\mathrm{cmH}_{2} \mathrm{O}$ & $10(8-12)$ & $10(8-12)$ & 0.476 \\
\hline Plateau pressure, $\mathrm{cmH}_{2} \mathrm{O}$ & $23(20-26)$ & $28(20-28)$ & 0.144 \\
\hline Driving pressure, $\mathrm{cmH}_{2} \mathrm{O}$ & $12(10-15)$ & $18(17-18)$ & 0.001 \\
\hline Respiratory-system compliance ${ }^{* *}, \mathrm{~mL} / \mathrm{cmH}_{2} \mathrm{O}$ & $38(31-45)$ & $23(22-27)$ & 0.001 \\
\hline \multicolumn{4}{|l|}{ Hemodynamic parameters } \\
\hline Heart rate, bpm & $90(72-109)$ & $105(69-118)$ & 0.494 \\
\hline Mean arterial blood pressure, $\mathrm{mmHg}$ & $102(85-110)$ & $78(71-94)$ & $<0.001$ \\
\hline CVP, mmHg & $9(7-10)$ & $11(9-14)$ & 0.058 \\
\hline
\end{tabular}


Table 1 Characteristics, presentation and outcome of ventilated patients with COVID-19 and flu-related ARDS (Continued)

\begin{tabular}{|c|c|c|c|}
\hline & COVID-19 $(n=18)$ & Flu $(n=23)$ & $p$ value \\
\hline ACP (\%) & $3(17)$ & $11(48)$ & 0.051 \\
\hline Severe ACP (\%) & $1(5)$ & $8(35)$ & 0.054 \\
\hline LV failure & $3^{* * *}(17)$ & $14(61)$ & 0.009 \\
\hline Hypovolemia & $2(11)$ & $1(4)$ & 0.573 \\
\hline Hyperkinesia & $6(33)$ & $7(30)$ & 1.00 \\
\hline Normal hemodynamic profile & $8(44)$ & $5(22)$ & 0.179 \\
\hline \multicolumn{4}{|l|}{ Echocardiographic indices } \\
\hline Cardiac index ${ }^{* * *}\left(\mathrm{~L} / \mathrm{min} / \mathrm{m}^{2}\right)$ & $3.1(2.5-4.2)$ & $2.5(2.0-3.0)$ & 0.034 \\
\hline RVEDA/LVEDA & $0.55(0.37-0.60)$ & $0.70(0.54-0.80)$ & 0.021 \\
\hline RVFAC, \% & $46(35-50)$ & $33(24-39)$ & 0.002 \\
\hline TAPSE, mm & $25(23-29)$ & $18(16-22)$ & $<0.001$ \\
\hline Tricuspid S', cm/s & $16.0(15.0-20.5)$ & $12.2(11.0-13.4)$ & 0.005 \\
\hline TR peak velocity, $\mathrm{m} / \mathrm{s}$ & $3.2(2.9-3.6)$ & $2.9(2.4-3.2)$ & 0.113 \\
\hline IVC diameter, mm & $22(19-26)$ & $22(21-24)$ & 0.762 \\
\hline LVEF (\%) & $52(44-61)$ & $44(28-59)$ & 0.265 \\
\hline LVOT VTI, cm & $22(18-25)$ & $18(13-24)$ & 0.106 \\
\hline Mitral E/E' ratio & $7.3(6.5-10.9)$ & $7.8(6.1-10.6)$ & 0.730 \\
\hline \multicolumn{4}{|l|}{ Outcome } \\
\hline ICU mortality*****(\%) & $1(6)$ & $9(39)$ & 0.025 \\
\hline
\end{tabular}

Abbreviations: BMI body mass index, SAPSII Simplified Acute Physiology Score, SOFA Sepsis Organ Failure Assessment, AST aspartate aminotransferase, $A L T$ alanine aminotransferase, CPK creatinine phosphokinase, RR respiratory rate, $P E E P$ positive end-expiratory pressure, CVP central venous pressure, $A C P$ acute cor pulmonale, $L V$ left ventricle, RVEDA right ventricular end-diastolic area, LVEDA left ventricular end-diastolic area, RVFAC right ventricular fractional area change, TAPSE tricuspid annular plane systolic excursion, TR tricuspid regurgitation, IVC inferior vena cava, LVEF left ventricular ejection fraction, LVOT left ventricular outflow tract, VTI velocity-time integral, ICU intensive care unit

*One patient had anterior negative T-wave in the COVID-19 group; 2 patients had inferior negative T-wave, and 1 patient had anterior negative T-wave in the flu group [2]

${ }^{* *}$ Calculated as the tidal volume divided by the driving pressure (difference between the inspiratory plateau pressure and positive end-expiratory pressure)

${ }^{* * *}$ One patient was diagnosed with a Tako-tsubo syndrome during transesophageal echocardiography examination performed shortly after tracheal intubation, after 6 days of high-flow nasal cannula; full recovery of left ventricular systolic function was documented under mechanical ventilation 10 days later

****Measured using the Doppler method applied at the left ventricular outflow tract

*****As per April 24, with still 6 patients hospitalized in the intensive care unit, 5 of them being invasively ventilated

The prevalence of left ventricular (LV) failure $(3 / 18$ [17\%] vs. $14 / 23$ [61\%], $p=0.009)$, ACP (3/18 [17\%] vs. $11 / 23[48 \%], p=0.051)$, and severe ACP $(1 / 18$ [5.5\%] vs. $8 / 23[35 \%], p=0.054)$ was significantly lower in COVID-19 patients. Hypovolemic and hyperkinetic phenotypes were similarly observed in both groups (Table 1). Despite similar tidal volume and PEEP level, COVID-19 patients had significantly higher P/F ratio and respiratory-system compliance, and lower driving pressure than flu patients (Table 1). Pulmonary embolism was identified in none of COVID-19 patients but in one flu patient with ACP. COVID-19 patients with ACP tended to exhibit lower respiratory-system compliance $(34,32$, and $30 \mathrm{~mL} /$ $\mathrm{cmH}_{2} \mathrm{O}$ ) when compared to others $(40$ [31-45] mL/ $\mathrm{cmH}_{2} \mathrm{O}$ ). Hemodynamic profile of COVID-19 patients remained stable during the first 3 days of ICU stay (Table 2).
The higher prevalence of LV failure and lower cardiac index in patients with flu-related ARDS is presumably related to septic cardiomyopathy since they sustained associated septic shock more frequently than COVID-19 patients. Depressed indices of RV systolic function and elevated central venous pressure reflecting systemic venous congestion reflect the higher prevalence of RV failure in flu ARDS patients (Table 1). This presumably results from the lower $\mathrm{P} / \mathrm{F}$, higher driving pressure, and lower respiratory-system compliance observed in this group. COVID-19 patients with ACP tended to have lower respiratory-system compliance than their counterparts, presumably due to distinct ARDS phenotypes [6]. This pilot study is limited by its small sample size and the retrospective comparison with historical flu-related ARDS patients.

This first study assessing hemodynamically ventilated COVID-19 patients with TEE shows a lower 
Table 2 Evolution of hemodynamic profile during daily transesophageal echocardiography assessments of COVID-19 patients ventilated for ARDS

\begin{tabular}{|c|c|c|c|c|}
\hline & Day $1(\boldsymbol{n}=18)$ & Day $2(\boldsymbol{n}=10)$ & Day $3(\boldsymbol{n}=12)$ & $p$ value \\
\hline \multicolumn{5}{|l|}{ Respiratory parameters } \\
\hline $\mathrm{PaO}_{2} / \mathrm{FiO}_{2}$ & $130(81-217)$ & $128(100-210)$ & $137(98-187)$ & 0.066 \\
\hline $\mathrm{PaCO}_{2}, \mathrm{mmHg}$ & $44(33-51)$ & $50(32-56)$ & $47(37-57)$ & 0.964 \\
\hline RR, breaths/min & $24(22-27)$ & $27(20-28)$ & $24(24-30)$ & 0.651 \\
\hline PEEP, $\mathrm{cmH}_{2} \mathrm{O}$ & $10(8-12)$ & $10(8-13)$ & $10(10-12)$ & 0.444 \\
\hline Plateau pressure, $\mathrm{cmH}_{2} \mathrm{O}$ & $23(20-26)$ & $22(18-27)$ & $24(21-27)$ & 0.127 \\
\hline Driving pressure, $\mathrm{cmH}_{2} \mathrm{O}$ & $12(10-15)$ & $11(9-12)$ & $13(11-17)$ & 0.368 \\
\hline Tidal volume, $\mathrm{mL} / \mathrm{kg}$ & $5.2(4.5-6.2)$ & $5.3(4.6-6.6)$ & $5.5(4.3-6.7)$ & 0.210 \\
\hline Respiratory-system compliance*, $\mathrm{mL} / \mathrm{cmH}_{2} \mathrm{O}$ & $38(31-45)$ & $33(33-53)$ & $37(28-45)$ & 0.692 \\
\hline \multicolumn{5}{|l|}{ Hemodynamic parameters } \\
\hline Heart rate, bpm & $90(72-109)$ & $93(78-107)$ & $98(89-104)$ & 0.368 \\
\hline $\mathrm{CVP}, \mathrm{mmHg}$ & $9(7-10)$ & $7(6-10)$ & $9(5-13)$ & 0.678 \\
\hline Mean blood pressure, mmHg & $102(85-110)$ & $105(87-110)$ & 95 (84-109) & 0.102 \\
\hline Lactate, $\mathrm{mmol} / \mathrm{L}$ & $1.17(0.89-1.57)$ & $1.85(1.24-3.01)$ & $1.62(1.49-1.95)$ & 0.264 \\
\hline \multicolumn{5}{|l|}{ Echocardiography indices } \\
\hline Cardiac index $\left(L / \mathrm{min} / \mathrm{m}^{2}\right)^{* *}$ & $3.1(2.5-4.2)$ & $2.8(2.6-3.9)$ & $4.1(3.2-4.8)$ & 0.115 \\
\hline RVEDA/LVEDA & $0.55(0.37-0.60)$ & $0.53(0.35-0.66)$ & $0.55(0.48-0.58)$ & 0.549 \\
\hline RVFAC, \% & $46(35-50)$ & $40(33-46)$ & $40(32-58)$ & 0.821 \\
\hline TAPSE, mm & $25(23-29)$ & $24(20-28)$ & $25(23-28)$ & 0.368 \\
\hline Tricuspid $\mathrm{S}^{\prime}, \mathrm{cm} / \mathrm{s}$ & $16.0(15.0-20.5)$ & $16.1(14.0-18.1)$ & $16.8(14.9-19.9)$ & 0.867 \\
\hline TR peak velocity, $\mathrm{m} / \mathrm{s}$ & $3.2(2.9-3.6)$ & $3.0(2.7-3.7)$ & $3.6(2.4-3.9)$ & 0.060 \\
\hline IVC diameter, mm & $22(19-26)$ & $24(14-30)$ & $22(17-24)$ & 1.000 \\
\hline LVEF, \% & $52(44-61)$ & $46(41-64)$ & $55(49-60)$ & 0.549 \\
\hline
\end{tabular}

Abbreviations: $R R$ respiratory rate, PEEP positive end-expiratory pressure, CVP central venous pressure, RVEDA right ventricular end-diastolic area, LVEDA left ventricular end-diastolic area, RVFAC right ventricular fractional area change, TAPSE tricuspid annular plane systolic excursion, TR tricuspid regurgitation, IVC inferior vena cava, LVEF left ventricular ejection fraction

${ }^{*}$ Calculated as the tidal volume divided by the driving pressure (difference between the inspiratory plateau pressure and positive end-expiratory pressure) ${ }^{*}$ Measured using the Doppler method applied at the left ventricular outflow tract

prevalence of LV and RV failure than in flu-related ARDS patients. Whether herein reported cardiovascular phenotypes are influenced by the type of COVID-19 ARDS remains to be determined [6]. These preliminary data warrant confirmation in large-scale multicenter cohorts.

\section{Abbreviations}

ACP: Acute cor pulmonale; ARDS: Acute respiratory distress syndrome; COVID-19: Coronavirus disease 2019; ICU: Intensive care unit; LV: Left ventricle; RV: Right ventricle; SAPS II: Simplified acute physiology score II; SOFA: Sepsis-related organ failure assessment

\section{Acknowledgements}

N/A

\section{Authors' contributions}

$B E, M G, A L F$, and PV included patients, analyzed the data, and drafted the manuscript. NM and TL collected and analyzed the data and reviewed the manuscript. All authors read and approved the final version of the manuscript.
Funding

None

\section{Availability of data and materials}

N/A

Ethics approval and consent to participate

Local Ethical Committee approval \#368-2020-24, which waived the need for informed consent. All patients agreed on the use of anonymized information as per the French law on the General Data Protection Regulation (GDPR).

\section{Consent for publication}

N/A

\section{Competing interests}

None

\section{Author details}

${ }^{1}$ Medical-Surgical Intensive Care Unit, Dupuytren Teaching Hospital, 87000 Limoges, France. ${ }^{2}$ Inserm CIC 1435, Dupuytren Teaching Hospital, 87000 Limoges, France. ${ }^{3}$ Emergency Department, Dupuytren Teaching Hospital, 87000 Limoges, France. ${ }^{4}$ Faculty of Medicine, University of Limoges, 87000 Limoges, France. ${ }^{5}$ Inserm UMR 1092, Dupuytren Teaching Hospital, 87000 Limoges, France. ${ }^{6}$ Réanimation Polyvalente, CHU Dupuytren, 2 Avenue Martin Luther king, 87042 Limoges, France. 
Received: 28 April 2020 Accepted: 7 May 2020

Published online: 18 May 2020

\section{References}

1. Yang X, Yu Y, Xu J, Shu H, Xia J, Liu H, Wu Y, Zhang L, Yu Z, Fang M, Yu T, Wang Y, Pan S, Zou X, Yuan S, Shang Y. Clinical course and outcomes of critically ill patients with SARS-CoV-2 pneumonia in Wuhan, China: a singlecentered, retrospective, observational study. Lancet Respir Med. 2020. https://doi.org/10.1016/S2213-2600(20)30079-5.

2. Hendren NS, Drazner MH, Bozkurt B, Cooper LT. Description of the acute COVID-19 cardiovascular syndrome. Circulation. 2020. https://doi.org/10. 1161/CIRCULATIONAHA.120.047349.

3. Li J-W, Han T-W, Woodward M, Anderson CS, Zhou H, Chen Y-D, Neal B. The impact of 2019 novel coronavirus on heart injury: a systematic review and meta-analysis. Prog Cardiovasc Dis. 2020. https://doi.org/10.1016/j.pcad.2020. 04.008.

4. Geri G, Vignon P, Aubry A, Fedou AL, Charron C, Silva S, Repessé X, VieillardBaron A. Cardiovascular clusters in septic shock combining clinical and echocardiographic parameters: a post hoc analysis. Intensive Care Med. 2019;45(5):657-67. https://doi.org/10.1007/s00134-019-05596-z.

5. Mekontso Dessap A, Boissier F, Charron C, Bégot E, Repessé X, Legras A, Brun-Buisson C, Vignon P, Vieillard-Baron A. Acute cor pulmonale during protective ventilation for acute respiratory distress syndrome: prevalence, predictors, and clinical impact. Intensive Care Med. 2016;42(5):862-70. https://doi.org/10.1007/s00134-015-4141-2.

6. Gattinoni L, Chiumello D, Rossi S. COVID-19 pneumonia: ARDS or not? Crit Care. 2020;24(1):154. https://doi.org/10.1186/s13054-020-02880-z.

\section{Publisher's Note}

Springer Nature remains neutral with regard to jurisdictional claims in published maps and institutional affiliations.

\section{Ready to submit your research? Choose BMC and benefit from:}

- fast, convenient online submission

- thorough peer review by experienced researchers in your field

- rapid publication on acceptance

- support for research data, including large and complex data types

- gold Open Access which fosters wider collaboration and increased citations

- maximum visibility for your research: over $100 \mathrm{M}$ website views per year

At BMC, research is always in progress.

Learn more biomedcentral.com/submissions 